\title{
Formation of Molecular-Orbital Bands in a Twisted Hubbard Tube: Implications for Unconventional Superconductivity in $\mathrm{K}_{2} \mathrm{Cr}_{3} \mathrm{As}_{3}$
}

\author{
Hanting Zhong, ${ }^{1}$ Xiao-Yong Feng, ${ }^{1,2}$, 田 Hua Chen, ${ }^{3,4}$ and Jianhui Dai ${ }^{1,2, \oplus}$ \\ ${ }^{1}$ Condensed Matter Group, Department of Physics, \\ Hangzhou Normal University, Hangzhou 310036, China \\ ${ }^{2}$ Hangzhou Key Laboratory of Quantum Matter, Hangzhou Normal University, Hangzhou 310036, China \\ ${ }^{3}$ International Center for Quantum Materials and School of Physics, Peking University, Beijing 100871, China \\ ${ }^{4}$ Collaborative Innovation Center of Quantum Matter, Beijing 100871, China
}

\begin{abstract}
We study a twisted Hubbard tube modeling the $[\mathrm{CrAs}]_{\infty}$ structure of quasi-one-dimensional superconductors $A_{2} \mathrm{Cr}_{3} \mathrm{As}_{3}(A=\mathrm{K}, \mathrm{Rb}, \mathrm{Cs})$. The molecular-orbital bands emerging from the quasidegenerate atomic orbitals are exactly solved. An effective Hamiltonian is derived for a region where three partially filled bands intersect the Fermi energy. The deduced local interactions among these active bands show a significant reduction compared to the original atomic interactions. The resulting three-channel Luttinger liquid shows various interaction-induced instabilities including two kinds of spin-triplet superconducting instabilities due to gapless spin excitations, with one of them being superseded by the spin-density-wave phase in the intermediate Hund's coupling regime. The implications of these results for the alkali chromium arsenides are discussed.
\end{abstract}

PACS numbers: 71.10.Pm; 72.15.Nj; 74.20.Mn; 74.70.-b

Introduction.-Recently, the alkali chromium arsenides $A_{2} \mathrm{Cr}_{3} \mathrm{As}_{3}(A=\mathrm{K}, \mathrm{Rb}, \mathrm{Cs})$ have been found as a new family of inorganic quasi-one-dimensional (Q1D) superconductors with strong electron correlations $\stackrel{1-3}{-3}$ The basic building block of these compounds is the $[\mathrm{CrAs}]_{6}$ cluster consisting of two conjugated triangular complexes $[\mathrm{CrAs}]_{3}$ as shown schematically in Fig[1(a). They are aligned along the $\mathrm{c}$ axis forming a $[\mathrm{CrAs}]_{\infty}$ tube, and intercalated by $A^{+}$cations forming a hexagonal lattice. The density functional theory (DFT) calculations 4.5 predict a three-dimensional (3D) Fermi surface (FS) sheet ( $\gamma$ band) and two Q1D FS sheets ( $\alpha$ and $\beta$ bands), essentially due to the $\mathrm{Cr} 3 d$ electrons. The NMR experiment $\underline{\underline{6}}$ has revealed a power law behavior of the spin-lattice relaxation rate, manifesting the Luttinger liquid feature above $T_{c}$. The penetration depth measurement $\underline{\underline{7}}$ has evidenced a line nodal feature in the pairing state below $T_{c}$.

Because of the existing 3D $\gamma$ band, whether the superconductivity is solely originated from the Q1D structure of $A_{2} \mathrm{Cr}_{3} \mathrm{As}_{3}$ is uncertain. In fact the nearly isotropic 3D bulk CrAs compound shows superconductivity with $T_{c} \sim$ $2.2 \mathrm{~K}$ under pressure of $\sim 0.7 \mathrm{GPa} . \stackrel{8.9}{*}$ Interestingly, Zhou et al. pointed out that an $f$-wave pairing state could arise from the 3D band with a node line while a fully gapped $p$-wave pairing state could dominate at the Q1D band $\underline{\underline{10}}$ Such triplet superconductivity, with some variations in spatial symmetry $\stackrel{11}{ }$, could be driven by ferromagnetic fluctuations within the sublattice of $\mathrm{Cr}$ atoms ${ }^{4.11}$.

In order to understand the formation of the low energy bands, it is particularly important to understand the electronic property of a single fundamental $[\mathrm{CrAs}]_{\infty}$ tube. In this Letter, we model this system by a twisted Hubbard lattice composed of triangular complexes coupled along the $\mathrm{c}$ axis with the glide reflection symmetry as shown in Fig 1(b) $\stackrel{12}{=}$ In each unit cell there are six $\mathrm{Cr}$ atoms, each with five $3 d$ atomic orbitals (AOs). The influence (a)

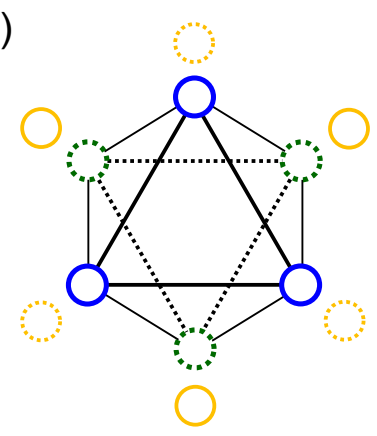

(b)

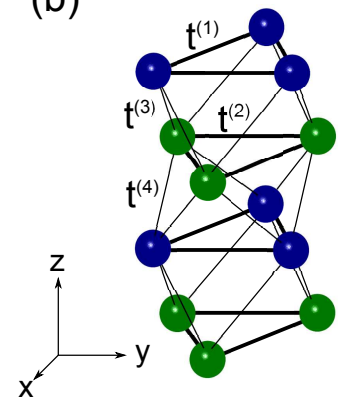

FIG. 1: (Color online) (a) A CrAs cluster in the $a b$ plane. The solid (dotted) circles connected by the solid (dotted) lines represent the $\mathrm{Cr}$ atoms in the first (second) triangle in a unit cell. The isolated outer solid (dotted) circles represent the As atoms in the corresponding planes. (b) A Q1D CrAs tube. The blue (green) filled circles represent the $\mathrm{Cr}$ atoms in each triangles. The As atoms are not shown.

of the As $4 p$ orbitals can be effectively accounted for the indirect hopping of $\mathrm{Cr}-3 d$ electrons. So the model involves thirty energy bands in total. In the realistic case, fortunately, only three partially filled bands are active in the low energy regime. We will explicitly show how these bands come from the molecular orbitals (MOs) of $[\mathrm{CrAs}]_{6}, \frac{40}{10}$. Our purpose is then to understand their cooperative low temperature physics within the Luttinger liquid approach. The proposed effective model is of interest in its own right as we shall explore in the following.

Model Hamiltonian. - The Hubbard model for a single [CrAs $]_{\infty}$ tube is expressed as $H=H_{0}+H_{\text {int }}$, where, $H_{0}$ represents the noninteracting part consisting of the tight-binding kinetic energy and the crystalline electric 
field (CEF) splitting,

$$
H_{0}=-\sum_{\mathbf{r} \mathbf{r}^{\prime} m m^{\prime} \sigma} t_{m m^{\prime}}^{\left(\mathbf{r}, \mathbf{r}^{\prime}\right)} d_{\mathbf{r} \sigma}^{\dagger(m)} d_{\mathbf{r}^{\prime} \sigma}^{\left(m^{\prime}\right)}+\sum_{\mathbf{r} m \sigma} E_{\mathbf{r} m} n_{\mathbf{r} \sigma}^{(m)}
$$

Here, $d_{\mathbf{r} \sigma}^{(m)}$ denotes the annihilation operator of $\mathrm{Cr} 3 d$ electrons at the site $\mathbf{r}$ with angular momentum $m=$ $0, \pm 1, \pm 2$, spin $\sigma=\uparrow, \downarrow . \quad n_{\mathbf{r} \sigma}^{(m)}$ and $\mathbf{S}_{\mathbf{r}}^{(m)}$ are the corresponding density and spin operators. The two twisted Cr triangles could have different $E_{\mathbf{r} m}=E_{m}^{(1)}, E_{m}^{(2)}$, accountable for the possible occupation difference ${ }^{\frac{5}{}}$, while

$$
\begin{aligned}
H_{\mathrm{int}}= & U \sum_{\mathbf{r} m} n_{\mathbf{r} \uparrow}^{(m)} n_{\mathbf{r} \downarrow}^{(m)}+\frac{2 U^{\prime}-J_{H}}{4} \sum_{\mathbf{r} m \neq m^{\prime} \sigma \sigma^{\prime}} n_{\mathbf{r} \sigma}^{(m)} n_{\mathbf{r} \sigma^{\prime}}^{\left(m^{\prime}\right)} \\
& -J_{H} \sum_{\mathbf{r} m \neq m^{\prime}} \mathbf{S}_{\mathbf{r}}^{(m)} \cdot \mathbf{S}_{\mathbf{r}}^{\left(m^{\prime}\right)} \\
& +J_{p} \sum_{\mathbf{r} m \neq m^{\prime}} d_{\mathbf{r} \uparrow}^{\dagger(m)} d_{\mathbf{r} \downarrow}^{\dagger(m)} d_{\mathbf{r} \downarrow}^{\left(m^{\prime}\right)} d_{\mathbf{r} \uparrow}^{\left(m^{\prime}\right)}
\end{aligned}
$$

represents the local interactions including the intraorbital Coulomb interaction $U$, the interorbital Coulomb interaction $U^{\prime}$, the Hund's coupling $J_{H}$, and the pair-hopping $J_{p}$, respectively.

There are four kinds of adjacent intraorbital hoppings $t_{m m}^{(i)} \equiv t_{m}^{(i)}(i=1-4)$, corresponding to the nearestneighbor (NN) sites in the first and second triangles, and those between the intracell and intercell triangles, respectively, as illustrated in Fig 1(b). Because of the metallic bonding among $\mathrm{Cr}$ atoms, the direct orbital mixings are relatively small, and the indirect hybridization is mainly bridged by the As $4 p$ orbitals. So it is legitimate to consider a simpler situation for the adjacent interorbital hopping: $t_{m m^{\prime}}^{(i)}=\eta t_{m}^{(i)} \delta_{|m|,\left|m^{\prime}\right|}$ for $m \neq m^{\prime}$, with $|\eta|<1$. In this situation, the atomic orbitals are quasidegenerate as the nonvanishing mix terms are isotropic in space $\frac{13}{}$. Finally, we include the next $\mathrm{NN}$ intraorbital hopping $t_{m}^{(5)}$ along the tube direction.

Molecular-orbital bands.-Denoting each site by $\mathbf{r}=(n, a, \xi)$, with $a=1,2,3$ being the location in the first $(\xi=1)$ or second $(\xi=2)$ triangles in the $n$th unit cell, it is convenient to introduce a base $d_{n}^{(m)}=$ $\left(d_{(n, 1,1)}^{(m)}, d_{(n, 1,2)}^{(m)}, d_{(n, 2,1)}^{(m)}, d_{(n, 2,2)}^{(m)}, d_{(n, 3,1)}^{(m)}, d_{(n, 3,2)}^{(m)}\right)^{T} \quad$ for the atomic $m$-orbital in the $n$th unit cell (the spin index $\sigma$ is implied). For $m=0$, this base accommodates a representation for the $C_{3}$ rotational symmetry, leading to six MOs corresponding to $E, E^{\prime}, A$, and $A^{\prime}$ states, respectively 14 . For $m= \pm 1$ or \pm 2 , we need to introduce a set of new base $\tilde{d}_{n}^{( \pm|m|)}=\frac{1}{\sqrt{2}}\left[d_{n}^{(m)} \pm d_{n}^{(-m)}\right]$. Thus for a single $[\mathrm{CrAs}]_{6}$ cluster, we have thirty MOs defined by $C_{n}^{(\tau)}=\left(\hat{R} \otimes \hat{Q}_{0}\right) \tilde{d}_{n}^{(\tau)}$ for $\tau=0$ (denoting $\left.\tilde{d}_{n}^{(0)} \equiv d_{n}^{(0)}\right), \pm 1$ and \pm 2 , respectively, with $\left(\omega=e^{i \varphi}, \varphi=2 \pi / 3\right)$

$$
\hat{R}=\frac{1}{\sqrt{3}}\left(\begin{array}{ccc}
1 & 1 & 1 \\
1 & \omega & \omega^{-1} \\
1 & \omega^{-1} & \omega
\end{array}\right), \hat{Q}_{0}=\frac{1}{\sqrt{2}}\left(\begin{array}{cc}
1 & -1 \\
1 & 1
\end{array}\right) .
$$

Note that the eigenstates of $\hat{R}$ with eigenvalues $\lambda_{1}=2$ and $\lambda_{2}=\lambda_{3}=-1$ constitute of representations $A$ and $E$ (or $A^{\prime}$ and $E^{\prime}$ ), respectively.

When the triangles are coupled along the $\mathrm{c}$ axis via the intercell hopping $t_{m m^{\prime}}^{(4)}$, we can extend $\hat{Q}_{0}$ to the momentum $k$-resolved matrix $\hat{Q}_{a}^{(\tau)}(k)$ so that the Bloch form $C_{k}^{(\tau)}=\left(c_{(k, 1,1)}^{(\tau)}, c_{(k, 1,2)}^{(\tau)}, c_{(k, 2,1)}^{(\tau)}, c_{(k, 2,2)}^{(\tau)}, c_{(k, 3,1)}^{(\tau)}, c_{(k, 3,2)}^{(\tau)}\right)^{T}$ is still a natural base diagonalizing $H_{0}$, leading to thirty MO bands labeled by the eigenenergies $\mathcal{E}_{(a, \xi)}^{(\tau)}(k)$. Here the subscript $\xi=1,2$ corresponds to the antibonding or bonding bands, respectively, due to the twisted structure. The explicit expressions of $\hat{Q}_{a}^{(\tau)}(k)$ and $\mathcal{E}_{(a, \xi)}^{(\tau)}(k)$, which also depend on the orbitals $\tau(=0, \pm 1, \pm 2)$ and $C_{3}$ eigenvalues $\lambda_{a}(a=1,2,3)$, are presented in the Supplemental Material $(\mathrm{SM})^{15}$. A set of subscripts $(a, \xi)$ determines the symmetry property of the corresponding MO bands.

We fitted the DFT band structure along the tube direction using the obtained $\mathrm{MO}$ bands within $\tau=0, \pm 2$, while the bands with $\tau= \pm 1$ are fairly away from the Fermi energy as revealed in the DFT calculations 4.5 . The three partially filled DFT bands, i.e., the 3D $\gamma$ band characterized mainly by the $d_{z^{2}}$ orbital $(m=0)$, the Q1D $\alpha$ and $\beta$ bands characterized mainly by the $d_{x y}$ and $d_{x^{2}-y^{2}}$ orbitals $(|m|=2)$, are all holelike near the $\Gamma$ point $(k=0)$ and electronlike near the $A$ point $(k=\pi)$. Therefore, the $\gamma$ band corresponds to the singlet MO band labeled by $(\tau=0, a=1, \xi=1)$. The $\alpha$ and $\beta$ bands, which are degenerate along the whole $\Gamma \rightarrow A$ direction, correspond to the doublet $\mathrm{MO}$ bands labeled by $(\tau=-2, a=2, \xi=2)$ and $(\tau=-2, a=3, \xi=2)$, respectively. The best fitting using $\mathcal{E}_{(1,1)}^{(0)}, \mathcal{E}_{(2,2)}^{(-2)}=\mathcal{E}_{(3,2)}^{(-2)}$ is shown in $\operatorname{Fig} 2^{15}$. Here, the tight-binding parameters are not uniquely determined because the number of these parameters exceeds eight necessary coefficients in the fitting. On the other hand, the precise values of the fitting parameters are not important in the present study. As we shall find later, only symmetry property of the MO bands and local interactions between them play a crucial role in the Luttinger liquid approach.

For simplicity, from now on, we shall use the band subscript $\nu(=1,2,3)$ to account for the three MO bands intersecting the Fermi energy. These active MO bands are associated with phases $\varphi_{\nu}=0,2 \pi / 3,-2 \pi / 3$, or chiralities $\vartheta_{\nu}=0,1$, and -1 , respectively. In the full 1D Brillouin zone, there are three pairs of Fermi points $\left(k_{F_{\nu}},-k_{F_{\nu}}\right)$, satisfying $0<k_{F_{1}}<k_{F_{2}}=k_{F_{3}}<\pi$, as schematically shown in the inset of Fig 2 By integrating out all inactive bands, we obtain the effective theory describing the low energy property of the active bands:

$$
H_{\mathrm{eff}}=\sum_{k \nu_{i} \sigma} \mathcal{E}_{\nu}(k) \hat{n}_{k \nu \sigma}+\sum_{n} H_{\mathrm{int}}^{(n)} .
$$

Where, $\hat{n}_{k \nu \sigma}=c_{k \nu \sigma}^{\dagger} c_{k \nu \sigma}$ is the density operator of electrons in the $\nu$ th MO band, $H_{\text {int }}^{(n)}$ the residual short-range 


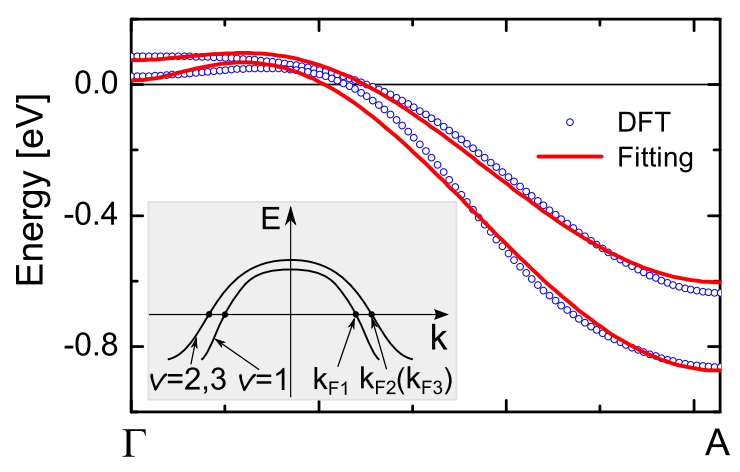

FIG. 2: (Color online) Fitting the band structure: The upper band is twofold degenerate. The lower inset is the schematic picture for the three partially filled bands with three pairs of Fermi points.

interactions in the MOs in the $n$th unit cell, given by

$$
\begin{aligned}
H_{\mathrm{int}}^{(n)} & =\sum_{\nu} \tilde{U}_{\nu} \hat{n}_{\nu \uparrow}(n) \hat{n}_{\nu \downarrow}(n)+\sum_{\nu \neq \nu^{\prime} \sigma \sigma^{\prime}} \tilde{U}_{\nu \nu^{\prime}} \hat{n}_{\nu \sigma}(n) \hat{n}_{\nu^{\prime} \sigma^{\prime}}(n) \\
& -\sum_{\nu \neq \nu^{\prime}} \tilde{J}_{\nu \nu^{\prime}} \hat{\mathbf{S}}_{\nu}(n) \cdot \hat{\mathbf{S}}_{\nu^{\prime}}(n) \\
& +\tilde{J}_{123}\left[c_{1 \uparrow}^{\dagger}(n) c_{1 \downarrow}^{\dagger}(n) c_{2 \downarrow}(n) c_{3 \uparrow}(n)+(2 \leftrightarrow 3)+\text { H.c. }\right] .
\end{aligned}
$$

In this expression, the electron annihilation operator in the $n$-th unit cell is defined by the Fourier transformation $c_{\nu \sigma}(n)=\frac{1}{\sqrt{2 \pi}} \sum_{k} e^{i k n c_{0}} c_{k \nu \sigma}$ (with $c_{0}$ the lattice spacing taken as unit). Only those terms preserving the neutrality condition $\sum_{\nu_{i}} \vartheta_{\nu_{i}}=0$ could survive. The matrix $\hat{Q}_{0}$ is used in deducing Eq.(5) as the short-range interactions are mainly due to the slowly varying part, leading to $\tilde{U}_{1}=U / 6, \tilde{U}_{2}=\tilde{U}_{3}=\left(U+U^{\prime}+J_{H}+J_{p}\right) / 12$; $\tilde{U}_{12}=\tilde{U}_{13}=U^{\prime} / 12-J_{H} / 24 ; \tilde{U}_{23}=\left(U+U^{\prime}+J_{H}+J_{p}\right) / 48 ;$ $\tilde{J}_{12}=\tilde{J}_{13}=J_{H} / 6 ; \tilde{J}_{23}=\left(U+U^{\prime}+J_{H}+J_{p}\right) / 12 ;$ and $\tilde{J}_{123}=J_{p} / 6$. The influence of inactive bands is mainly accounted to the renormalized tight-binding parameters.

The Luttinger liquid in the weak-coupling regime.We now take the continuous limit, linearize the active bands near the Fermi points, and decompose the electron operator into right and left moving components like $c_{\nu \sigma}(z) \approx e^{-i k_{F_{\nu}} z-i \varphi_{\nu}} L_{\nu \sigma}(z)+e^{+i k_{F_{\nu}} z+i \varphi_{\nu}} R_{\nu \sigma}(z)$. Here, $z=n c_{0}$ is the spatial coordinate along the tube direction, $R_{\nu \sigma}$ and $L_{\nu \sigma}$ represent the right and left moving fermions describing the low energy excitations near the Fermi points $\left(k_{F_{\nu}},-k_{F_{\nu}}\right)$ with linear dispersion $\pm v_{F_{\nu}} k$. The long-wavelength, low-energy effective Hamiltonian (density) is given by $\mathcal{H}_{\text {eff }}=\mathcal{H}_{0}+\mathcal{H}_{\text {int }}$, where $\mathcal{H}_{0}=$ $\sum_{\nu, \sigma}\left(i v_{F_{\nu}}\right)\left[R_{\nu \sigma}^{\dagger} \partial_{z} R_{\nu \sigma}-L_{\nu \sigma}^{\dagger} \partial_{z} L_{\nu \sigma}\right]$ is the kinetic part, and $\mathcal{H}_{\text {int }}$ includes various residual interactions which are usually expressed in terms of the $g$-ology $\frac{16,17}{}$. We shall assume the Fermi velocities $v_{F_{\nu}}$ to be the same as this does not influence the nature of superconductivity we concern. The corresponding one-loop renormalization group (RG) equations resemble those for three-leg Hubbard ladders $\frac{18,19}{19}$ or a variant of carbon nanotubes ${ }^{20}-22$. The instabilities of these RG equations are classified routinely: (i) the intraband instabilities as those developed in the single-channel Luttinger liquid ${ }^{23}$, and (ii) the interband instabilities as those developed in the two-channel band Luttinger liquid. Note that the three-band interaction in Eq.(5) does not lead to the peculiar three-band instability suggested in Ref. $\underline{22}$ as shown in the SM 15,24 . All these suggest the validity of the conventional bosonization approach based on spin-charge separation, where various ordering instabilities can be determined by Luttinger parameters. The new ingredients here are the peculiar symmetry surviving in the active MO bands and their dependence on local electron interactions.

The right- and left-moving fields are then expressed in terms of the charge fields $\left(\phi_{\nu, c}, \theta_{\nu, c}\right)$ and the spin fields $\left(\phi_{\nu, s}, \theta_{\nu, s}\right)($ for each $\nu=1,2,3)$ by

$$
\begin{aligned}
R_{\nu, \sigma}(z) & =\frac{F_{R, \nu \sigma}}{\sqrt{2 \pi c_{0}}} e^{i \sqrt{\pi / 2}\left(\theta_{c, \nu}+\sigma \theta_{s, \nu}-\phi_{c, \nu}-\sigma \phi_{s, \nu}\right)}, \\
L_{\nu, \sigma}(z) & =\frac{F_{L, \nu \sigma}}{\sqrt{2 \pi c_{0}}} e^{i \sqrt{\pi / 2}\left(\theta_{c, \nu}+\sigma \theta_{s, \nu}+\phi_{c, \nu}+\sigma \phi_{s, \nu}\right)} .
\end{aligned}
$$

The Klein factors $F_{R, \nu \sigma}$ and $F_{L, \nu \sigma}$ ensure the fermionic statistics between the right and left moving fermions. Next, in order to diagonalize the kinetic part, we need to introduce a set of new base

$$
\begin{aligned}
\tilde{\phi}_{\gamma, i} & =\eta_{\gamma, i}\left(q_{\gamma, i} \phi_{\gamma, 1}+\phi_{\gamma, 2}+\phi_{\gamma, 3}\right), \\
\tilde{\phi}_{\gamma, 3} & =\frac{1}{\sqrt{2}}\left(-\phi_{\gamma, 2}+\phi_{\gamma, 3}\right),
\end{aligned}
$$

where $\gamma=s, c, q_{\gamma, i}=-\frac{b_{\gamma}+(-1)^{i} \sqrt{8 a_{\gamma}^{2}+b_{\gamma}^{2}}}{2 a_{\gamma}}$ for $i=1,2$, $a_{c}=\frac{2 \tilde{U}_{12}}{\pi}, b_{c}=\frac{2 \tilde{U}_{23}}{\pi}, a_{s}=-\frac{\tilde{J}_{12}}{2 \pi}, b_{s}=-\frac{\tilde{J}_{23}}{2 \pi}, \eta_{\gamma, i}$ are the normalization constants. Similar relationships apply to the fields $\theta_{\gamma, i}$ and $\tilde{\theta}_{\gamma, i}$ for $i=1,2,3$. Hence, we arrive at the following three-channel Tomonaga-Luttinger liquid Hamiltonian:

$$
\tilde{H}_{0}=\int d z \sum_{i=1,2,3, \gamma=s, c}\left[\frac{v_{F}}{2}\left(\nabla \tilde{\theta}_{\gamma, i}\right)^{2}+\lambda_{\gamma, i}\left(\nabla \tilde{\phi}_{\gamma, i}\right)^{2}\right]
$$

where, $\lambda_{\gamma, i}=t_{\gamma}+\frac{1}{2}\left[b_{\gamma}-(-1)^{i} \sqrt{8 a_{\gamma}^{2}+b_{\gamma}^{2}}\right]$ for $i=1,2$, and $\lambda_{\gamma, 3}=t_{\gamma}-b_{\gamma}, t_{c}=\frac{v_{F}}{2}+\frac{\tilde{U}_{1}}{2 \pi}, t_{s}=\frac{v_{F}}{2}-\frac{\tilde{U}_{1}}{2 \pi}$. Therefore, the Luttinger parameters are obtained explicitly by

$$
\begin{aligned}
& K_{c, i}=\left[1+\frac{U}{4 \pi v_{F}}-\frac{(-1)^{i}}{12 \pi v_{F}} \sqrt{8\left(2 U-5 J_{H}\right)^{2}+U^{2}}\right]^{-\frac{1}{2}}, \\
& K_{s, i}=\left[1-\frac{U}{4 \pi v_{F}}-\frac{(-1)^{i}}{12 \pi v_{F}} \sqrt{8 J_{H}^{2}+U^{2}}\right]^{-\frac{1}{2}}
\end{aligned}
$$

for the channels $i=1,2$, respectively, and $K_{c, 3}=K_{s, 3}=$ 1 for the third channel $i=3$. Here, we have adopted the conventional relations $J_{p}=J_{H}$ and $U^{\prime}=U-2 J_{H}$, reflecting the rotational symmetry of the original AOs $\$ 25$.

Now since $U>0$ and $J_{H}>0$, one can find that: (i) $K_{c, 1}<1$ in the entire region and $K_{s, 1}<1$ only when $U<$ 


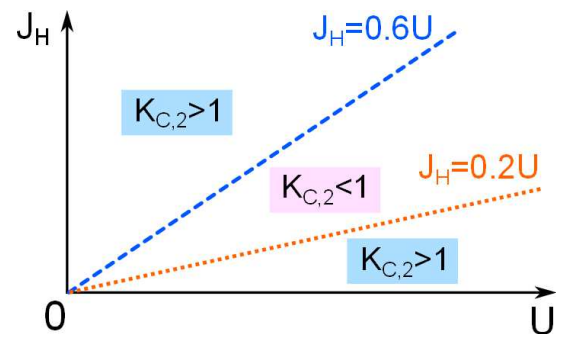

FIG. 3: (Color online) Luttinger parameters for the channel-2: $K_{s, 2}>1$ everywhere, and $K_{c, 2}<1$ only in the intermediate regime between the lines $J_{H}=0.2 U$ and $J_{H}=0.6 U$.

$J_{H}$; (ii) $K_{c, 2}<1$ in the region $0.2 U<J_{H}<0.6 U$ and $K_{s, 2}>1$ in the entire region. Specifically, in the physically relevant regime, $U>J_{H}$, the spin excitations are always gapless, so $K_{s, i}$ could be fixed to the unit due to the spin-SU(2) symmetry. Because $K_{c, 1}<1$, the channel"1" is in the spin-density-wave (SDW) phase $\frac{16,17}{10}$. The channel-" 3" involves the antibonding of the MO bands $\nu=2,3$ as shown in Eq.(17). In this channel both spin and charge excitations are critical. Because of the absence of a spin gap, the dominating superconducting instability is the interband spin triplet pairing ${ }^{16,17}$, driven by the interband scattering between the two Q1D $\alpha$ and $\beta$ bands. The intriguing case is the channel-" 2 ", whose property depends on the ratio $J_{H} / U$. We plot in Fig. 3 the phase diagram determined by the Luttinger parameters in this channel. We find that $K_{c, 2}>1$ in the regimes separated by the orange-dotted and blue-dashed lines, respectively. In these two separated regimes, the dominating instability is still the spin-triplet pairing ${ }^{16.17}$. But in the intermediate regime where $K_{c, 2}<1$, the SDW instability dominates. It should be noticed that in either case where the interband triplet superconducting instabilities dominate, the spin-singlet superconducting instability is the subdominating instability ${ }^{17,26}$.

In order to see whether the above Luttinger liquid results are robust against deviations from the atomic orbital rotational symmetry, we have also considered a small deviation $\Delta U$ away from the rotational symmetry by assuming $U^{\prime}=U+\Delta U-2 J_{H}$. As shown in the SM, the channel-3 is still in the critical phase and the role of $\Delta U$ is to modify the value of $\mathrm{U}$ in a simple manner so that the results remain unchanged 15 .

Summary and discussions. - We have focused on the microscopic formation of the MO bands in a twisted Hubbard tube capturing the Q1D nature of $\mathrm{K}_{2} \mathrm{Cr}_{3} \mathrm{As}_{3}$, a new Q1D multiorbital superconducting molecular crystal with the moderate Coulomb interaction and Hund's coupling. A three-channel Tomonaga-Luttinger liquid Hamiltonian describing the low energy physics of the three active MO bands ( the $\alpha, \beta$, and $\gamma$ bands) is then derived, showing possible unconventional triplet superconducting instabilities within a reasonable range of interaction parameters.

The conclusions and implications of our study are compared with the previous studies $\underline{10}, 11$ where a phenomeno- logical 3D Hubbard model for the three active MO bands was proposed based on the elegant symmetry argument 10 and investigated by the random phase approximation 10.11 and the mean field treatment $\underline{\underline{11}}$. First, the twisted structure of the Q1D $[\mathrm{CrAs}]_{\infty}$ tube showing the extended glide reflection symmetry in accordance with the $C_{3}$ group is explored in our approach so that the symmetry property of all the thirty MO bands (including the three active MO bands) could be identified. Second, the interactions among the three active MO bands are derived from the microscopic atomic Hubbard interactions, different from those proposed phenomenologically. Third, the three diagonal channels in our Tomonaga-Luttinger Hamiltonian are superpositions of the original DFT bands, in contrast to the random phase approximation approach and the mean field treatment.

We found two kinds of spin-triplet pairing instabilities emerging out from two of the three channels. One involves the Q1D $\alpha$ and $\beta$ bands, another involves all three bands. In the Luttinger liquid approach, triplet pairing instabilities are due to gapless spin excitations for $U>J_{H}$ and $K_{c}>1$ in the corresponding channels. The ferromagnetic correlation within the sublattice of $\mathrm{Cr}$ atoms ${ }^{4.11}$, though possible, is not a prerequisite of the triplet states. We also found an intermediate regime $0.2<J_{H} / U<0.6$ where the SDW phase emerges. Our solution is sensitive to the symmetry or regularity of the two conjugated $\mathrm{Cr}$ triangles, seemingly consistent with the recent hydrostatic and uniaxial pressure experimental study 27 . The exact mapping from the AOs to MO bands will also pave the way for further investigations on related effects such as the spin-orbit coupling within a microscopic framework.

As the present study is limited to the Q1D case, the spatial symmetry of the superconducting pairing states is not specified. However, the actual 3D superconductivity can be perceived based on the Q1D physics because the identification of the three low energy MO bands is robust owing to the same symmetry argument. The local interactions among the MO bands are similar to those in the Q1D case. If the local atomic interactions are estimated as those in other Cr-based oxides, like $\mathrm{SrCrO}_{3}{ }^{28}$, one has $U \sim 2.7 \pm 0.5 \mathrm{eV}, J_{H} \sim 0.42 \pm 0.1 \mathrm{eV}$, and $J_{H} / U \sim 0.16$, then in the Q1D case the channel-2 is in the triplet phase but close to the SDW low boundary $J_{H} / U=0.2$ shown in Fig 3 , The corresponding residual MO interactions $\tilde{U}$ and $J$ in the $3 \mathrm{D}$ case are significantly suppressed, but the ratio $\tilde{J} / \tilde{U}$ enhanced ${ }^{15}$, corresponding to the regime with small $\tilde{U}$ but relatively large $\tilde{J} / \tilde{U}$ in $\operatorname{Ref} \frac{10}{10}$, where the triplet $f_{y\left(3 x^{2}-y^{2}\right)}$ pairing state is favored. Of course, we have not considered the long-range Coulomb interaction and the electron-phonon coupling, the suppression of residual MO interactions should necessitate further investigations on these influences.

Finally, a more intriguing issue is the possible dimensional crossover from Q1D to 3D which could be tuned by either chemical substitution 2,3 or physical pressure 27,29 . On one hand, one of the three active bands, correspond- 
ing to $\nu=1$, evolves with the intertube hopping and crossovers to the $3 \mathrm{D} \gamma$ band which could lead to the line nodal feature. Meanwhile, the $(\alpha, \beta)$ bands could remain in Q1D because the intertube hopping among the AOs with $m= \pm 2$ is reasonably small. On the other hand, the Q1D superconducting instability can lead to a true long-range order when the intertube hopping is taken into account. Recall that the interband triplet pairing instability in the channel- 2 is driven not only by the $\gamma$ band, but also by the $(\alpha, \beta)$ bands. Consequently, the spin-triplet pairing instability in the channel-2 involves both the 3D and Q1D bands. As such a 3D pairing state could emerge from a normal state of an essentially Q1D Luttinger liquid characteristic, a scenario which is likely consistent with available experiments. It is desirable to investigate the related crossover behavior in this class of materials in the future.

We are grateful to Chao Cao, Guanghan Cao, and Yi Zhou for stimulating discussions. This work was supported in part by the NSF of China (under Grants No. 11274084, No. 11304071, and No. 11474082).
* Electronic address: fxyong@163.com

$\dagger$ Electronic address: daijh@zju.edu.cn

1 J.-K. Bao et al., Phys. Rev. X 5, 011013 (2015).

2 Z.-T. Tang et al., Phys. Rev. B 91, 020506(R) (2015).

3 Z.-T. Tang et al., Sci. China Mater. 58, 16 (2015).

${ }^{4}$ H. Jiang, G. Cao, and C. Cao, Sci. Rep. 5, 16054 (2015).

${ }^{5}$ X. Wu et al., Chin. Phys. Lett. 32, 057401 (2015).

${ }^{6}$ H.Z. Zhi et al., Phys. Rev. Lett. 114, 147004 (2015).

7 G.M. Pang et al., Phys. Rev. B 91, 220502(R) (2015).

${ }^{8}$ W. Wei et al., Nat. Commun. 5, 5508(2014).

9 H. Kotegawa, N. Nakahara, H. Tou, and H. Sugawara, J. Phys. Soc. Jpn. 83, 093702 (2014).

10 Y. Zhou, C. Cao, and F.C. Zhang, arXiv:1502.03928.

11 X. Wu et al., Phys. Rev. B 92, 104511 (2015).

12 The glide reflection symmetry is exact when the two conjugated triangles are equivalent and the corresponding hopping parameters and the CEF splittings in the Hamiltonian Eq.(1) are the same.

13 The assumption of quasidegenerate AOs leads to a fixed AO content of each MO band along the $\Gamma \rightarrow A$ direction up to a rotation around the $z$ axis. The variation of the AO contents does not alter the symmetry property and local interactions of the MOs.

14 R.B. Tao, Group Theory in Physics (Higher Education Press, Beijing, China, 2011).

15 See Supplemental Material at http://link.aps.org/supplemental/10.1103/physRevLett.115.227001] for the detailed solutions of the tight-binding Hamiltonian, supplemental discussions on the band structure fitting, a comparison with previous theoretical studies, the one-loop RG equations, and the Luttinger parameters away from the condi- tion of atomic orbital rotational symmetry.

16 J. Solyom, Adv. Phys. 28, 201 (1979).

17 T. Giamarchi, Quantum Physics in One Dimension (Oxford University Press, Oxford, UK, 2003).

18 E. Arrigoni, Phys. Lett. A 215, 91 (1996).

19 H.-H. Lin, L. Balents, and M.P.A. Fisher, Phys. Rev. B 56, 6569 (1997).

20 Y.A. Krotov, D.-H. Lee, and S.G. Louie, Phys. Rev. Lett. 78, 4245 (1997).

21 J. Gonzalez and E. Perfetto, Phys. Rev. B 72, 205406 (2005).

22 D. Carpentier and E. Orignac, Phys. Rev. B 74, 085409 (2006).

23 In the Luttinger liquid approach the electronic interactions among various MO bands impact the kinetic Hamiltonian after bosonization, leading to reconstruction of the original DFT bands. So we shall use the concept "channel" for the three diagonal quasiparticle bands as defined in Eq.(7).

24 The three-band instability discussed in Ref. ${ }^{22}$ may appear when the electron-phonon coupling is relatively larger than the pair hopping or the Hund's coupling.

25 C. Castellani, C.R. Natoli, and J. Ranninger, Phys. Rev. B 18, 5001 (1978).

26 The present study does not exclude the possibility of a spin singlet pairing due to the critical nature of the channel 3 .

27 Z. Wang et al., arXiv:1502.04304v1.

28 L. Vaugier, H. Jiang, and S. Biermann, Phys. Rev. B 86, 165105 (2012).

29 T. Kong, S.L. Budko, and P.C. Canfield, Phys. Rev. B 91, 020507 (2015). 


\section{"Formation of Molecular-Orbital Bands in a Twisted Hubbard Tube: Implications for Unconventional Superconductivity in $\mathrm{K}_{2} \mathrm{Cr}_{3} \mathrm{As}_{3} "$}

By: Hanting Zhong, Xiao-Yong Feng, Hua Chen, and Jianhui Dai

\section{SUPPLEMENTAL MATERIAL}

This is the Supplemental Material (SM) for our paper titled "Formation of Molecular-Orbital Bands in a Twisted Hubbard Tube: Implications for Unconventional Superconductivity in $\mathrm{K}_{2} \mathrm{Cr}_{3} \mathrm{As}_{3}$ ",$\frac{1}{1}$ In this SM, we provide the detailed solution of the tight-binding Hamiltonian as well as some supplemental discussions on various related issues, including the band structure fitting, a comparison with previous theoretical studies, the one-loop RG equations, and the Luttinger parameters away from the condition of atomic orbital rotational symmetry.

\section{A. Solution of orbital quasi-degenerate tight-binding Hamiltonian and fitting the band structure}

The tight-binding Hamiltonian discussed in the main text is given by

$$
\begin{aligned}
H_{0}= & -\sum_{n, \sigma} \sum_{m, m^{\prime}} \sum_{I \neq I^{\prime}}\left\{t_{m m^{\prime}}^{(1)} d_{n I \sigma}^{\dagger(m)} d_{n I^{\prime} \sigma}^{\left(m^{\prime}\right)}+t_{m m^{\prime}}^{(2)} d_{n \bar{I} \sigma}^{\dagger(m)} d_{n \bar{I}^{\prime} \sigma}^{\left(m^{\prime}\right)}+t_{m m^{\prime}}^{(3)} d_{n I \sigma}^{\dagger(m)} d_{n \bar{I}^{\prime} \sigma}^{\left(m^{\prime}\right)}+t_{m m^{\prime}}^{(4)} d_{n I \sigma}^{\dagger(m)} d_{n+1 \bar{I}^{\prime} \sigma}^{\left(m^{\prime}\right)}\right\} \\
& +\sum_{n, \sigma} \sum_{m, m^{\prime}} \sum_{I} t_{m m^{\prime}}^{(5)}\left\{d_{n I \sigma}^{\dagger(m)} d_{n+1 I \sigma}^{\left(m^{\prime}\right)}+d_{n \bar{I} \sigma}^{\dagger(m)} d_{n+1 \bar{I} \sigma}^{\left(m^{\prime}\right)}\right\}+\sum_{n, \sigma} \sum_{m} \sum_{I}\left\{E_{m} n_{n I \sigma}^{(m)}+\bar{E}_{m} n_{n \bar{I} \sigma}^{(m)}\right\}
\end{aligned}
$$

Where, $d_{n I \sigma}^{(m)}$ annihilates a Cr $3 d$-electron moving along the $z$-axis at the $n$-unit cell, with spin polarization $\sigma(=\uparrow, \downarrow)$, orbital component $m(=0, \pm 2)$, intra-triangle location $I(=A, B, C)$, as well as the conjugate triangle location $\bar{I}(=$ $\bar{A}, \bar{B}, \bar{C})$. Here, the site locations correspond to $(a, \xi)$ introduced in the main text as: $A=(1,1), B=(2,1), C=(3,1)$, $\bar{A}=(1,2), \bar{B}=(2,2), \bar{C}=(3,2)$. The notation $(a, \xi)$ introduced in the main text is explicit to accommodate the group representation, while $(A, B, C)$ or $(\bar{A}, \bar{B}, \bar{C})$ are more transparent. Either notations will be used for convenience in this SM. The hopping parameters $t_{m m^{\prime}}^{(i)}(i=1,2,3$ and 4$)$ are those for the n.n. sites shown in Fig.1. In the present orbital quasi-degenerate model, these intraorbital and interorbital hopping parameters satisfy the relationship $t_{m m^{\prime}}^{(i)}=\eta t_{m}^{(i)} \delta_{|m|\left|m^{\prime}\right|}$ for $m \neq m^{\prime}$ with the ratio $|\eta|<1$ ( In the following we take $\eta>0$ without losing the generality). $t_{m m^{\prime}}^{(5)}=t_{m}^{(5)} \delta_{m m^{\prime}}$ is the next nearest neighbor (intraorbital) hopping along the tube direction. The CEF term is given by $E_{m}$ (or $\left.\bar{E}_{m}\right)$, with $n_{n I \sigma}^{(m)}$ (or $n_{n \bar{I} \sigma}^{(m)}$ ) being the corresponding density operators.

In order to solve the non-interacting Hamiltonian for a single cluster [CrAs $]_{6}$ in the n-th unit cell, $H_{0, n}$, we introduce $d_{n}^{(m)}=\left(d_{(n, A)}^{(m)}, d_{(n, \bar{A})}^{(m)}, d_{(n, B)}^{(m)}, d_{(n, \bar{B})}^{(m)}, d_{(n, C)}^{(m)}, d_{(n, \bar{C})}^{(m)}\right)^{T}$ for each $m=0, \pm 1, \pm 2$ (the spin index $\sigma$ is implied). Because of orbital mixing between $m= \pm 1$ or \pm 2 , we need to introduce another set of base $\tilde{d}_{n}^{( \pm|m|)}=\frac{1}{\sqrt{2}}\left[d_{n}^{(m)} \pm d_{n}^{(-m)}\right]$. Then all thirty atomic orbitals (MOs) in a unit cell can be described by $\tilde{d}_{n}^{(\tau)}$, with $\tau=0, \pm 1, \pm 2\left(\operatorname{denote} \tilde{d}_{n}^{(\tau=0)} \equiv d_{n}^{(m=0)}\right.$ ) The MOs of the n-th cluster, $C_{n}^{(\tau)}=\left(c_{(n, 1,1)}^{(\tau)}, c_{(n, 1,2)}^{(\tau)}, c_{(n, 2,1)}^{(\tau)}, c_{(n, 2,2)}^{(\tau)}, c_{(n, 3,1)}^{(\tau)}, c_{(n, 3,2)}^{(\tau)}\right)^{T}$, are defined as the base which diagonalizes Hamiltonian $H_{0, n}=\sum_{\tau} C_{n}^{\dagger(\tau)} H_{0, n}^{(\tau)} C_{n}^{(\tau)}$. Here, $H_{0, n}^{(\tau)}=\operatorname{diag}\left\{E_{(a, \xi)}^{(\tau)}\right\}$ is a diagonal matrix with eigenvalues $E_{(a, \xi)}^{(\tau)}$. The MOs can be obtained by $C_{n}^{(\tau)}=\left(\hat{R} \otimes \hat{Q}_{0}\right) \tilde{d}_{n}^{(\tau)}$ with $\omega=e^{i \varphi}, \varphi=2 \pi / 3, \hat{R}$ and $\hat{Q}_{0}$ are given in Eq.(3) in 
the main text. Explicitly, we have ${ }^{2}$

$$
\begin{aligned}
& c_{(n, 1,1)}^{(\tau)}=\frac{1}{\sqrt{6}}\left(\tilde{d}_{(n, A)}^{(\tau)}+\tilde{d}_{(n, \bar{A})}^{(\tau)}+\tilde{d}_{(n, B)}^{(\tau)}+\tilde{d}_{(n, \bar{B})}^{(\tau)}+\tilde{d}_{(n, C)}^{(\tau)}+\tilde{d}_{(n, \bar{C})}^{(\tau)}\right), \\
& c_{(n, 1,2)}^{(\tau)}=\frac{1}{\sqrt{6}}\left(\tilde{d}_{(n, A)}^{(\tau)}-\tilde{d}_{(n, \bar{A})}^{(\tau)}+\tilde{d}_{(n, B)}^{(\tau)}-\tilde{d}_{(n, \bar{B})}^{(\tau)}+\tilde{d}_{(n, C)}^{(\tau)}-\tilde{d}_{(n, \bar{C})}^{(\tau)}\right), \\
& c_{(n, 2,1)}^{(\tau)}=\frac{1}{\sqrt{6}}\left(\tilde{d}_{(n, A)}^{(\tau)}+\tilde{d}_{(n, \bar{A})}^{(\tau)}+\omega \tilde{d}_{(n, B)}^{(\tau)}+\omega \tilde{d}_{(n, \bar{B})}^{(\tau)}+\omega^{-1} \tilde{d}_{(n, C)}^{(\tau)}+\omega^{-1} \tilde{d}_{(n, \bar{C})}^{(\tau)}\right), \\
& c_{(n, 2,2)}^{(\tau)}=\frac{1}{\sqrt{6}}\left(\tilde{d}_{(n, A)}^{(\tau)}-\tilde{d}_{(n, \bar{A})}^{(\tau)}+\omega \tilde{d}_{(n, B)}^{(\tau)}-\omega \tilde{d}_{(n, \bar{B})}^{(\tau)}+\omega^{-1} \tilde{d}_{(n, C)}^{(\tau)}-\omega^{-1} \tilde{d}_{(n, \bar{C})}^{(\tau)}\right), \\
& c_{(n, 3,1)}^{(\tau)}=\frac{1}{\sqrt{6}}\left(\tilde{d}_{(n, A)}^{(\tau)}+\tilde{d}_{(n, \bar{A})}^{(\tau)}+\omega^{-1} \tilde{d}_{(n, B)}^{(\tau)}+\omega^{-1} \tilde{d}_{(n, \bar{B})}^{(\tau)}+\omega \tilde{d}_{(n, C)}^{(\tau)}+\omega \tilde{d}_{(n, \bar{C})}^{(\tau)}\right), \\
& c_{(n, 3,2)}^{(\tau)}=\frac{1}{\sqrt{6}}\left(\tilde{d}_{(n, A)}^{(\tau)}-\tilde{d}_{(n, \bar{A})}^{(\tau)}+\omega^{-1} \tilde{d}_{(n, B)}^{(\tau)}-\omega^{-1} \tilde{d}_{(n, \bar{B})}^{(\tau)}+\omega \tilde{d}_{(n, C)}^{(\tau)}-\omega \tilde{d}_{(n, \bar{C})}^{(\tau)}\right)
\end{aligned}
$$

In order to solve the whole tight-binding Hamiltonian $H_{0}$, we need to introduce $d_{k}^{(m)}=$ $\left(d_{(k, A)}^{(m)}, d_{(k, \bar{A})}^{(m)}, d_{(k, B)}^{(m)}, d_{(k, \bar{B})}^{(m)}, d_{(k, C)}^{(m)}, d_{(k, \bar{C})}^{(m)}\right)^{T}$, and the corresponding $\tilde{d}_{k}^{(\tau)}$ in the momentum space. Then, the Hamiltonian can be diagonalized by $C_{k}^{(\tau)}=\left(c_{(k, 1,1)}^{(\tau)}, c_{(k, 1,2)}^{(\tau)}, c_{(k, 2,1)}^{(\tau)}, c_{(k, 2,2)}^{(\tau)}, c_{(k, 3,1)}^{(\tau)}, c_{(k, 3,2)}^{(\tau)}\right)^{T}$, in the form of $H_{0}=\sum_{\tau, k} C_{k}^{\dagger(\tau)} H_{0, k}^{(\tau)} C_{k}^{(\tau)}$. Here, $H_{0, k}^{(\tau)}=\operatorname{diag}\left\{\mathcal{E}_{(a, \xi)}^{(\tau)}(k)\right\}$ is the diagonal matrix with eigenvalues $\mathcal{E}_{(a, \xi)}^{(\tau)}(k)$ given by

$$
\mathcal{E}_{(a, \xi)}^{(\tau)}(k)=-\frac{\lambda_{a}^{(\tau)}}{2}\left[\epsilon_{(a, 1)}^{(\tau)}+\epsilon_{(a, 2)}^{(\tau)}+(-1)^{\xi} \sqrt{\left(\epsilon_{(a, 1)}^{(\tau)}-\epsilon_{(a, 2)}^{(\tau)}\right)^{2}+4 \rho_{\tau}^{2}}\right]
$$

In above, $k$ is the crystal momentum along the tube direction, $\lambda_{a}^{(\tau)}=[1+\operatorname{sign}(\tau)|\eta|] \lambda_{a}$ for $a=1,2,3$, with $\lambda_{1}=2$ and $\lambda_{2}=\lambda_{3}=-1$ the eigenvalues of $\hat{R}, \epsilon_{(a, \xi)}^{(\tau)}=t_{\tau}^{(\xi)}+\frac{2 t_{\tau}^{(5)} \cos k-E_{\tau}^{(\xi)}-\mu}{\lambda_{a}^{(\tau)}}, \rho_{\tau}=\left|t_{\tau}^{(3)}+t_{\tau}^{(4)} e^{i k}\right|$, and $\mu$ the chemical potential.

Accordingly, the electron operators in MO bands are given by $C_{k}^{(\tau)}=\hat{R} \otimes \operatorname{diag}\left\{\hat{Q}_{a}^{(\tau)}(k)\right\} \tilde{d}_{k}^{(\tau)}$. Here, $\operatorname{diag}\left\{\hat{Q}_{a}^{(\tau)}(k)\right\}$ is a direct product of sub-matrices $\hat{Q}_{a}^{(\tau)}(k)$ defined for each eigenstates $\lambda_{a}$ of the $C_{3}$ rotation as given by

$$
\hat{Q}_{a}^{(\tau)}(k)=\left(\begin{array}{cc}
\cos \alpha_{(a, 1)}^{(\tau)} & \sin \alpha_{(a, 1)}^{(\tau)} e^{-i \theta_{\tau}} \\
\sin \alpha_{(a, 2)}^{(\tau)} e^{i \theta_{\tau}} & \cos \alpha_{(a, 2)}^{(\tau)}
\end{array}\right),
$$

with $\tan \theta_{a \tau}=\frac{t_{\tau}^{(4)} \sin k}{t_{\tau}^{(3)}+t_{\tau}^{(4)} \cos k}, \cos \alpha_{(a, \xi)}^{(\tau)}=\frac{1}{\sqrt{1+\left(\Delta_{(a, \xi)}^{(\tau)}\right)^{2}}}$, and $\Delta_{(a, \xi)}^{(\tau)}=\frac{\mathcal{E}_{(a, \xi)}^{(\tau)}-\epsilon_{(a, \xi)}^{(\tau)}}{\rho_{a \tau}}$

The Fourier transformation of $C_{k}^{(\tau)}$ back to the spatial space does not return exactly, though similar, to the forms as defined by Eq.(S2-S7), because the corresponding coefficients in each terms are now $k$-dependent due to the intercell coupling along the tube direction. This feature will in general lead to various long-range electron correlations among the MOs of different unit cells. However, upon summation over the whole all unit cells, the slowly varying terms dominate the contributions. So as long as only the local interactions of MO bands are concerned, $\hat{Q}_{0}$ or Eqs.(S2-S7) can be used to deduce these interactions as given in the next section.

In order to fit the DFT band structure, we re-express the eigenvalues in a more explicit form

$$
\begin{aligned}
\mathcal{E}_{(a, \xi)}^{(\tau)}(k)= & -2 t_{\tau}^{(5)} \cos k+\mu+\frac{E_{\tau}+\bar{E}_{\tau}}{2} \\
& -\frac{[1+\operatorname{sign}(\tau) \eta] \lambda_{a}}{2}\left\{t_{\tau}^{(1)}+t_{\tau}^{(2)}+(-1)^{\xi} \sqrt{\left(t_{\tau}^{(1)}-t_{\tau}^{(2)}-\frac{E_{\tau}-\bar{E}_{\tau}}{[1+\operatorname{sign}(\tau) \eta] \lambda_{a}}\right)^{2}+4\left|t_{\tau}^{(3)}+t_{\tau}^{(4)} e^{i k}\right|^{2}}\right\}
\end{aligned}
$$

As explained in the main text, we fit the three active DFT bands $\alpha, \beta$, and $\gamma$ along the $\Gamma$-A direction ( the tube direction or the c-axis $)^{3.4}$. The non-degenerated $\gamma$ band is contributed mainly from the $d_{z^{2}}$ orbital with $m=0$, is fitted by the singlet MO band indexed by $(\tau=0, a=1, \xi=1)$, with the energy $\mathcal{E}_{1,1}^{(0)}(k)$,

$$
\mathcal{E}_{(\gamma)}(k)=-2 t_{0}^{(5)} \cos k+\mu+\frac{E_{0}+\bar{E}_{0}}{2}-t_{0}^{(1)}-t_{0}^{(2)}+\sqrt{\left(t_{0}^{(1)}-t_{0}^{(2)}-\frac{E_{0}-\bar{E}_{0}}{2}\right)^{2}+4\left|t_{0}^{(3)}+t_{0}^{(4)} e^{i k}\right|^{2}} .
$$


The $\alpha$ - and $\beta$-bands, which are degenerate along the $\Gamma$-A direction, are fitted by the MO bands indexed by $(\tau=$ $-2, a=2, \xi=2)$ and $(\tau=-2, a=3, \xi=2)$, with the energy $\mathcal{E}_{(2,2)}^{(-2)}(k)=\mathcal{E}_{(3,2)}^{(-2)}(k)$

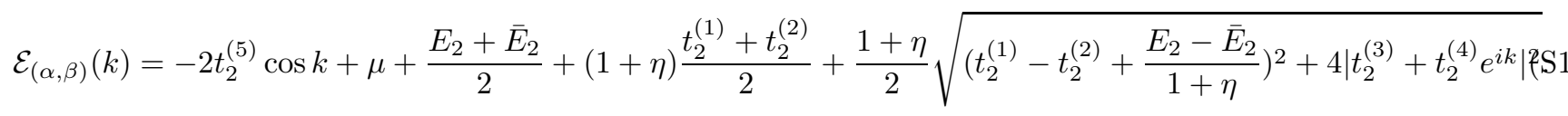

Both Eqs. $(\mathrm{S} 11, \mathrm{~S} 12)$ take the form as $\mathcal{E}(k) \propto A_{\tau}+B_{\tau} \cos k+\sqrt{C_{\tau}+D_{\tau} \cos ^{2} \frac{k}{2}}$, each with four independent coefficients $A_{\tau}, B_{\tau}, C_{\tau}$, and $D_{\tau}$. For the $\gamma$-band, $A_{0}=\mu+\frac{E_{0}+\bar{E}_{0}}{2}-t_{0}^{(1)}-t_{0}^{(2)}, B_{0}=-2 t_{0}^{(5)}, C_{0}=\left(t_{0}^{(1)}-t_{0}^{(2)}-\frac{E_{0}-\bar{E}_{0}}{2}\right)^{2}+4\left(t_{0}^{(3)}-t_{0}^{(4)}\right)^{2}$, $D_{0}=16 t_{0}^{(3)} t_{0}^{(4)}$. The best fitting is given by $A_{0}=-1.306 \mathrm{eV}, B_{0}=1.049 \mathrm{eV}, C_{0}=2.194 \mathrm{eV}$, and $D_{0}=-2.121 \mathrm{eV}$. For the $\alpha$ - and $\beta$-bands, $A_{2}=\mu+\frac{E_{2}+\bar{E}_{2}}{2}+\frac{1+\eta}{2}\left(t_{2}^{(1)}+t_{2}^{(2)}\right), B_{2}=-2 t_{2}^{(5)}, C_{2}=\frac{(1+\eta)^{2}}{4}\left[\left(t_{2}^{(1)}-t_{2}^{(2)}-\frac{E_{2}-\bar{E}_{2}}{2}\right)^{2}+4\left(t_{2}^{(3)}-t_{2}^{(4)}\right)^{2}\right]$, $D_{2}=4(1+\eta)^{2} t_{2}^{(3)} t_{2}^{(4)}$. The best fitting is given by $A_{2}=-1.500 \mathrm{eV}, B_{2}=0.962 \mathrm{eV}, C_{2}=3.450 \mathrm{eV}$, and $D_{2}=-3.074$ $\mathrm{eV}$. The negative $D_{\tau}$ implies opposite signs of $t_{\tau}^{(3)}$ and $t_{\tau}^{(4)}$. The relatively large value of $C_{\tau}$ implies a sizable difference in length or electron occupation between the two conjugated triangles as already indicated in the DFT calculations .3 .4

Of course, the above fitting is by no means rigorous, given the fact that the renormalization effect may be not adequately accounted in the DFT band structure. It is also possible to fit the DFT band structure within a reasonable approximation by other sets of parameters. For instance, we can use relatively smaller parameters $B_{(\tau)}$ and $C_{(\tau)}$, but positive $D_{\tau}$, the overall lineshape and band width are still closed to the DFT results. In comparison with the bare tight-binding parameters these fitting parameters should be all effective after renormalization. As far as the three active MO bands $\alpha, \beta$ and $\gamma$ are concerned, the fitting formulae involve eight independent coefficients. Because the total number of free tight-binding parameters used in fitting exceeds eight, we cannot determine these parameters uniquely. On the other hand, the precise values of these parameters are not important in our present study. As we have shown in the main text, only the symmetry property of the active MOs and local interactions between them play the most crucial role in the resultant Luttinger liquid theory.

\section{B. Molecular orbital interaction parameters and comparison with previous theoretical studies}

In the main text of this paper, we have considered the microscopic atomic orbital(AO) Hubbard model with the local intraorbital Coulomb interaction $U$ and interorbital Coulomb interaction $U^{\prime}$, Hund's coupling $J_{H}$, and pair hopping $J_{p}$, among all five atomic 3 d-orbitals $m=0, \pm 1, \pm 2$. Explicitly, the interaction matrices take the following forms

$$
\left(\begin{array}{ccccc}
U & U^{\prime} & U^{\prime} & U^{\prime} & U^{\prime} \\
U^{\prime} & U & U^{\prime} & U^{\prime} & U^{\prime} \\
U^{\prime} & U^{\prime} & U & U^{\prime} & U^{\prime} \\
U^{\prime} & U^{\prime} & U^{\prime} & U & U^{\prime} \\
U^{\prime} & U^{\prime} & U^{\prime} & U^{\prime} & U
\end{array}\right), \quad\left(\begin{array}{ccccc}
0 & J_{H} & J_{H} & J_{H} & J_{H} \\
J_{H} & 0 & J_{H} & J_{H} & J_{H} \\
J_{H} & J_{H} & 0 & J_{H} & J_{H} \\
J_{H} & J_{H} & J_{H} & 0 & J_{H} \\
J_{H} & J_{H} & J_{H} & J_{H} & 0
\end{array}\right), \quad\left(\begin{array}{cccccc}
0 & J_{p} & J_{p} & J_{p} & J_{p} \\
J_{p} & 0 & J_{p} & J_{p} & J_{p} \\
J_{p} & J_{p} & 0 & J_{p} & J_{p} \\
J_{p} & J_{p} & J_{p} & 0 & J_{p} \\
J_{p} & J_{p} & J_{p} & J_{p} & 0
\end{array}\right) .
$$

Using the inverse mapping from the MOs to AOs, various two-particle interactions among the MOs are induced by the above local AO interactions. In general, the induced MO interactions are non-local due to the inter-cell hopping. As far as the local MO interactions are focused, the matrix $\hat{Q}_{0}$ or Eqs.(S2-S7) can be used in deducing the local MO interactions given in Eq.(5) in the main text, as the short-range interactions are mainly due to the slowly-varying part. Such the local MO interactions depend on local AO interactions via various products taking the forms like

$$
\begin{gathered}
d_{(n, a, \xi), \sigma_{1}}^{\dagger\left(\tau_{1}\right)} d_{(n, a, \xi), \sigma_{2}}^{\dagger\left(\tau_{2}\right)} d_{(n, a, \xi), \sigma_{3}}^{\left(\tau_{3}\right)} d_{(n, a, \xi), \sigma_{4}}^{\left(\tau_{4}\right)} \\
=\sum_{a_{i}, \xi_{i}}\left[\hat{Q}_{0}^{-1} \otimes \hat{R}^{-1}\right]_{\left(\tau_{1}, a_{1}, \xi_{1}\right)}^{\dagger} \cdot\left[\hat{Q}_{0}^{-1} \otimes \hat{R}^{-1}\right]_{\left(\tau_{2}, a_{2}, \xi_{2}\right)}^{\dagger} \cdot\left[\hat{Q}_{0}^{-1} \otimes \hat{R}^{-1}\right]_{\left(\tau_{3}, a_{3}, \xi_{3}\right)} \cdot\left[\hat{Q}_{0}^{-1} \otimes \hat{R}^{-1}\right]_{\left(\tau_{4}, a_{4}, \xi_{4}\right)} \\
C_{\left(n, a_{1}, \xi_{1}\right), \sigma_{1}}^{\dagger\left(\tau_{1}\right)} C_{\left(n, a_{2}, \xi_{2}\right), \sigma_{2}}^{\dagger\left(\tau_{2}\right)} C_{\left(n, a_{3}, \xi_{3}\right), \sigma_{3}}^{\left(\tau_{3}\right)} C_{\left(n, a_{4}, \xi_{4}\right), \sigma_{4}}^{\left(\tau_{4}\right)} .
\end{gathered}
$$

In the previous section, by solving the tight-binding Hamiltonian and fitting the DFT band structure, the three active molecular orbital bands $\nu=1,2,3$ are identified. Based on the symmetry argument, $\nu=1$ corresponds to the $\gamma$-band with $m=0$, denoted by the molecular orbital electron annihilation operator $c_{1}(n) \equiv c_{(n, 1,1)}^{(0)}($ with $\tau=0, a=1, \xi=1)$ in Eq.(S2). While $\nu=2$ and $\nu=3$ correspond to the $\alpha$ - and $\beta$-bands with $m= \pm 2$, denoted by the molecular orbital electron annihilation operator $c_{2} \equiv c_{(n, 2,2)}^{(-2)}($ with $\tau=-2, a=2, \xi=2)$ and $c_{3} \equiv c_{(n, 3,2)}^{(-2)}($ with 
$\tau=-2, a=3, \xi=2)$ in Eqs.(S5) and (S7), respectively. After some tedious but straightforward algebras thanks to symmetry properties of the matrices $\hat{R}$ and $\hat{Q}_{0}$, the following interaction matrices for various two-particle Coulomb interactions and Hund's couplings for the above three active molecular orbitals are deduced:

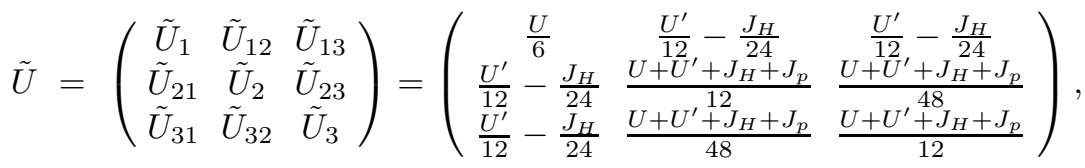

$$
\begin{aligned}
& \tilde{J}=\left(\begin{array}{ccc}
0 & \tilde{J}_{12} & \tilde{J}_{13} \\
\tilde{J}_{21} & 0 & \tilde{J}_{23} \\
\tilde{J}_{31} & \tilde{J}_{32} & 0
\end{array}\right)=\left(\begin{array}{ccc}
0 & \frac{J_{H}}{6} & \frac{J_{H}}{6} \\
\frac{J_{H}}{6} & 0 & \frac{U+U^{\prime}+J_{H}+J_{p}}{12} \\
\frac{J_{H}}{6} & \frac{U+U^{\prime}+J_{H}+J_{p}}{12} & 0
\end{array}\right) .
\end{aligned}
$$

In addition, a three-band interaction term

$$
\tilde{J}_{123}\left[c_{1 \uparrow}^{\dagger}(n) c_{1 \downarrow}^{\dagger}(n) c_{2 \downarrow}(n) c_{3 \uparrow}(n)+(2 \leftrightarrow 3)+\text { h.c. }\right]
$$

emerges, with $\tilde{J}_{123}=J_{p} / 6$. Note that this peculiar interaction is absent in the previous theoretical studies ${ }^{5}, 6$. However, as we shall show in the next section, this term does not influence the instabilities we concern.

Usually, we assume the orbital rotational symmetry of the interacting Hamiltonian in the atomic orbital Hubbard model, i.e., $U^{\prime}=U-2 J_{H}$ and $J_{p}=J_{H}{ }^{\underline{7}}$ For a given material, it is understood that values of these local interactions can be meaningfully determined when band structure calculations as using the constraint density functional approaches are implemented by a complete description of the corresponding atomic Hubbard model. So far such detailed calculations on the compound $\mathrm{K}_{2} \mathrm{Cr}_{3} \mathrm{As}_{3}$ are not yet available. However, these local atomic interactions can be inferred from those in other Cr-based oxides, like $\mathrm{SrCrO}_{3}$. According to $\mathrm{Ref} \stackrel{\underline{8}}{\text {, }}$, one has $U \sim 2.7 \mathrm{eV}, J_{H} \sim 0.42 \mathrm{eV}$ in $\mathrm{SrCrO}_{3}$. These values are relatively smaller than but still closed to those of $\mathrm{Fe}^{2+}$ systems like $\mathrm{SrFeO}_{2}$ or iron pnictides, where $U \sim 3-5$ $\mathrm{eV}, J_{H} \sim 0.50-0.70 \mathrm{eV} \underline{\underline{9}}$ The ratio $J_{H} / U$ of these systems does not change too much. Hence as a rough estimate, we assume the similar values of $\mathrm{SrCrO}_{3}$ for the present compound, obtaining the local molecular orbital interaction matrices from Eqs.(S15,S16):

$$
\tilde{U} \approx\left(\begin{array}{ccc}
0.45 & 0.14 & 0.14 \\
0.14 & 0.45 & 0.11 \\
0.14 & 0.11 & 0.45
\end{array}\right), \quad \tilde{J} \approx\left(\begin{array}{ccc}
0 & 0.07 & 0.07 \\
0.07 & 0 & 0.45 \\
0.07 & 0.45 & 0
\end{array}\right) .
$$

Notice that it is always possible for these values to be fluctuated within $20 \%$ or even more for a given material by using different approaches. With this understanding, it is interesting to compare our results with those in Refs $\underline{\underline{5} .6}$. In our Q1D Luttinger theory, while the channel "3" is always critical for a much wider regime $U>J_{H}$, the channel "2" is expected to be located at the spin triplet phase because $J_{H} / U \sim 0.16$. However, this triplet state should close to the border of the SDW phase, $J_{H} / U=0.2$, as shown in Fig. 3 in the main text.

In Ref $\underline{\underline{5}}$, the following interaction matrices for phenomenological molecular bands are introduced:

$$
\tilde{U}_{\mathrm{Z}}=\left(\begin{array}{ccc}
U_{1} & U_{2} & U_{2}^{\prime} \\
U_{2} & U_{1} & U_{2}^{\prime} \\
U_{2}^{\prime} & U_{2}^{\prime} & U_{1}^{\prime}
\end{array}\right), \quad \tilde{J}_{\mathrm{Z}}=\left(\begin{array}{ccc}
0 & J & J^{\prime} \\
J & 0 & J^{\prime} \\
J^{\prime} & J^{\prime} & 0
\end{array}\right), \quad \tilde{J}_{p}=\tilde{J}_{\mathrm{Z}}
$$

while in Ref.

$$
\tilde{U}_{\mathrm{W}}=\left(\begin{array}{ccc}
U & V & V \\
V & U & V \\
V & V & U
\end{array}\right), \quad \tilde{J}_{\mathrm{W}}=\left(\begin{array}{ccc}
0 & J_{H} & J_{H} \\
J_{H} & 0 & J_{H} \\
J_{H} & J_{H} & 0
\end{array}\right), \quad \tilde{J}_{p}=\tilde{J}_{\mathrm{W}}
$$

Here, we use the same notations introduced in Refs $\underset{5}{\underline{5}}$, respectively. The relationships $U_{1}^{\prime}=U_{1}, U_{2}^{\prime}=U_{2}$, and $0.5<J^{\prime} / J<2.0$ were used in calcuations ${ }^{5}$. It should be understood that (i) the parameters used in these studies are for the molecular orbital bands; (ii) they do not rigorously correspond to our interaction matrices given by Eqs.(S15S17). Nevertheless, we may expect that if the largest elements in the interaction matrices $\tilde{U}$ and $\tilde{J}$ dominate in the random phase approximation (RPA) approach, the values of atomic interactions estimated in our case correspond to the case of $U_{1}=0.45, J=0.45$, and $J / U_{1}=1$ in Ref.

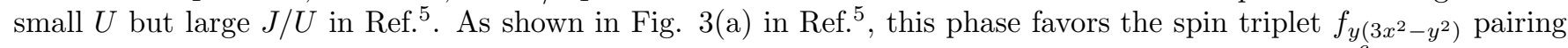
state. Notice that these values are out of the calculated regime of the phase diagram Fig (6) in Ref $\underline{6}$, but the same tendency is reached if this phase diagram is extended to large ration of $J / U$. 


\section{One-loop RG equations and absence of the three-band instability}

Owing to the symmetry between the MO bands $\nu=2$ and $\nu=3$, there are twelve permissible perturbations which can be classified in terms of the formal $g$-ology description ${ }^{10.11}$ using the same notation in ${ }^{12-14}$ :

$$
\begin{aligned}
\mathcal{H}_{\text {int }} & =g^{(1)} \sum_{\sigma \sigma^{\prime}} R_{1 \sigma}^{\dagger} L_{1 \sigma} L_{1 \sigma^{\prime}}^{\dagger} R_{1 \sigma^{\prime}} \\
& +g^{(2)} \sum_{\sigma \sigma^{\prime}} R_{1 \sigma}^{\dagger} R_{1 \sigma} L_{1 \sigma^{\prime}}^{\dagger} L_{1 \sigma^{\prime}} \\
& -g_{1}^{(1)} \sum_{\sigma \sigma^{\prime}} R_{2 \sigma}^{\dagger} R_{2 \sigma^{\prime}} L_{3 \sigma^{\prime}}^{\dagger} L_{3 \sigma}+(2 \leftrightarrow 3) \\
& -g_{1}^{(2)} \sum_{\sigma \sigma^{\prime}} R_{2 \sigma}^{\dagger} L_{2 \sigma^{\prime}} L_{3 \sigma^{\prime}}^{\dagger} R_{3 \sigma}+(2 \leftrightarrow 3) \\
& +g_{2}^{(1)} \sum_{\sigma \sigma^{\prime}} R_{2 \sigma}^{\dagger} L_{2 \sigma} L_{3 \sigma^{\prime}}^{\dagger} R_{3 \sigma^{\prime}}+(2 \leftrightarrow 3) \\
& +g_{2}^{(2)} \sum_{\sigma \sigma^{\prime}} R_{2 \sigma}^{\dagger} R_{2 \sigma} L_{3 \sigma^{\prime}}^{\dagger} L_{3 \sigma^{\prime}}+(2 \leftrightarrow 3) \\
& +g_{4}^{(1)} \sum_{\sigma \sigma^{\prime}} R_{2 \sigma}^{\dagger} L_{2 \sigma} L_{2 \sigma^{\prime}}^{\dagger} R_{2 \sigma^{\prime}}+(2 \leftrightarrow 3) \\
& +g_{4}^{(2)} \sum_{\sigma \sigma^{\prime}} R_{2 \sigma}^{\dagger} R_{2 \sigma} L_{2 \sigma^{\prime}}^{\dagger} L_{2 \sigma^{\prime}}+(2 \leftrightarrow 3) \\
& -f^{(1)} \sum_{\sigma \sigma^{\prime}}\left[R_{1 \sigma}^{\dagger} R_{1 \sigma^{\prime}} L_{2 \sigma^{\prime}}^{\dagger} L_{2 \sigma}+(2 \leftrightarrow 3)\right]+(R \leftrightarrow L) \\
& +f^{(2)} \sum_{\sigma \sigma^{\prime}}\left[R_{1 \sigma}^{\dagger} R_{1 \sigma} L_{2 \sigma^{\prime}}^{\dagger} L_{2 \sigma^{\prime}}+(2 \leftrightarrow 3)\right]+(R \leftrightarrow L) \\
& +u \sum_{\sigma \sigma^{\prime}}\left[R_{1 \sigma}^{\dagger} L_{1 \sigma^{\prime}}^{\dagger} L_{2 \sigma^{\prime}} R_{3 \sigma}+(2 \leftrightarrow 3)\right]+h . c . \\
& +v \sum_{\sigma \sigma^{\prime}}\left[R_{1 \sigma}^{\dagger} L_{1 \sigma^{\prime}}^{\dagger} R_{2 \sigma^{\prime}} L_{3 \sigma}+(2 \leftrightarrow 3)\right]+h . c . .
\end{aligned}
$$

In this notation, the degeneracy between the MO bands $\nu=2$ and $\nu=3$ are explicit. The one-loop renormalization group (RG) equations for these coupling constants evolving with increasing scaling parameter $l$ are of common type, given by $12-14$ :

$$
\begin{aligned}
\partial_{l} \tilde{g}^{(1)} & =-2\left(\tilde{g}^{(1)}\right)^{2}-4 \tilde{u} \tilde{v} \\
\partial_{l} \tilde{g}^{(2)} & =-\left(\tilde{g}^{(1)}\right)^{2}-2 \tilde{u}^{2}-2 \tilde{v}^{2} \\
\partial_{l} \tilde{g}_{1}^{(1)} & =-2\left(\tilde{g}_{1}^{(1)}\right)^{2}-\tilde{g}_{1}^{(2)} \tilde{g}_{2}^{(1)}-2 \tilde{u} \tilde{v} \\
\partial_{l} \tilde{g}_{1}^{(2)} & =-2 \tilde{g}_{1}^{(1)} \tilde{g}_{2}^{(1)}-\tilde{g}_{1}^{(2)} \tilde{g}_{2}^{(2)}+\tilde{g}_{1}^{(2)} \tilde{g}_{4}^{(2)}-\tilde{u}^{2}-\tilde{v}^{2} \\
\partial_{l} \tilde{g}_{2}^{(1)} & =-2 \tilde{g}_{1}^{(1)} \tilde{g}_{1}^{(2)}-2 \tilde{g}_{2}^{(1)} \tilde{g}_{2}^{(2)}+\tilde{g}_{1}^{(2)} \tilde{g}_{4}^{(1)}-4 \tilde{g}_{2}^{(1)} \tilde{g}_{4}^{(1)}+2 \tilde{g}_{2}^{(1)} \tilde{g}_{4}^{(2)}-2 \tilde{u} \tilde{v} \\
\partial_{l} \tilde{g}_{2}^{(2)} & =-\left(\tilde{g}_{1}^{(1)}\right)^{2}-\left(\tilde{g}_{1}^{(2)}\right)^{2}-\left(\tilde{g}_{2}^{(1)}\right)^{2}-\tilde{u}^{2}-\tilde{v}^{2} \\
\partial_{l} \tilde{g}_{4}^{(1)} & =2 \tilde{g}_{1}^{(2)} \tilde{g}_{2}^{(1)}-2\left(\tilde{g}_{2}^{(1)}\right)^{2}-2\left(\tilde{g}_{4}^{(1)}\right)^{2} \\
\partial_{l} \tilde{g}_{4}^{(2)} & =\left(\tilde{g}_{1}^{(2)}\right)^{2}-\left(\tilde{g}_{4}^{(1)}\right)^{2} \\
\partial_{l} \tilde{f}^{(1)} & =-2\left(\tilde{f}^{(1)}\right)^{2}+2 \tilde{u} \tilde{v}-2 \tilde{v}^{2} \\
\partial_{l} \tilde{f}^{(2)} & =-\left(\tilde{f}^{(1)}\right)^{2}+\tilde{u}^{2} \\
\partial_{l} \tilde{u} & =\left(2 \tilde{f}^{(2)}-\tilde{g}_{1}^{(2)}-\tilde{g}^{(2)}-\tilde{g}_{2}^{(2)}\right) \tilde{u}-\left(\tilde{f}^{(1)}+\tilde{g}_{1}^{(1)}+\tilde{g}_{2}^{(1)}\right) \tilde{v} \\
\partial_{l} \tilde{v} & =-\left(-2 \tilde{f}^{(1)}+\tilde{g}^{(1)}+\tilde{g}_{1}^{(1)}+\tilde{g}_{2}^{(1)}\right) \tilde{u}-\left(4 \tilde{f}^{(1)}-2 \tilde{f}^{(2)}+\tilde{g}_{1}^{(2)}+\tilde{g}^{(2)}+\tilde{g}_{2}^{(2)}\right) \tilde{v} .
\end{aligned}
$$




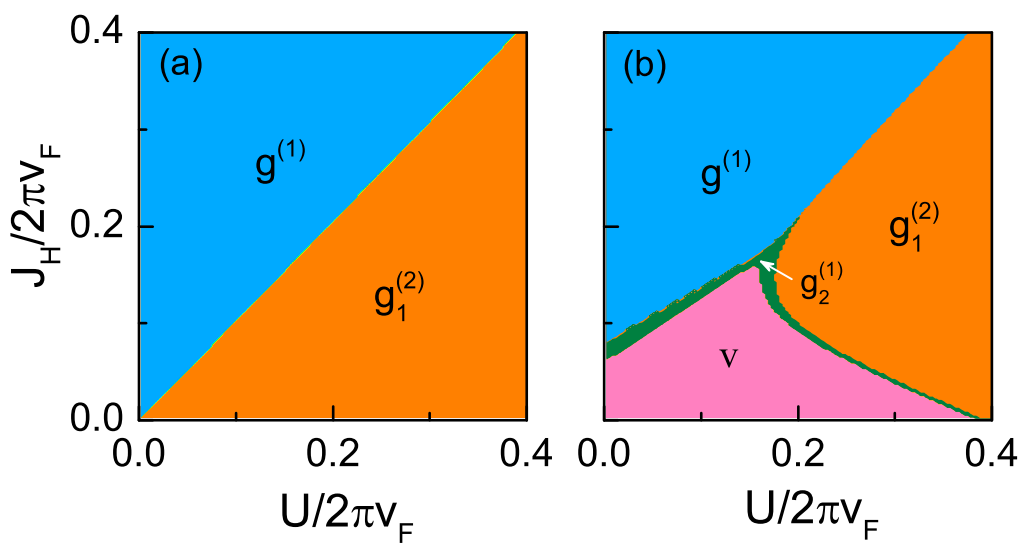

FIG. S1: The dominating perturbations in the one-loop RG equations. (a)All the initial parameters are given by Eqs.(S15$\mathrm{S} 17, \mathrm{~S} 22)$; (b) The initial values of $u$ and $v$ have the opposite sign using $v / 2 \pi v_{F}=J_{H} / 6-0.02$ and $u / 2 \pi v_{F}=J_{H} / 6$ for small $J_{H}$, while the other parameters are still given by Eqs. (S15-S17,S22).

In above, $\tilde{g} \equiv \frac{g}{2 \pi v_{F}}$, and

$$
\begin{array}{ll}
g^{(1)}=\tilde{U}_{1}, & g^{(2)}=\tilde{U}_{1}, \\
g_{1}^{(1)}=\tilde{J}_{23}, & g_{1}^{(2)}=\tilde{J}_{23}, \\
g_{2}^{(1)}=2 \tilde{U}_{23}+\frac{1}{2} \tilde{J}_{23}, & g_{2}^{(2)}=2 \tilde{U}_{23}+\frac{1}{2} \tilde{J}_{23}, \\
g_{4}^{(1)}=\tilde{U}_{2}, & g_{4}^{(2)}=\tilde{U}_{2}, \\
f^{(1)}=\tilde{J}_{12}, & f^{(2)}=2 \tilde{U}_{12}+\frac{1}{2} \tilde{J}_{12}, \\
u=\tilde{J}_{123}, & v=\tilde{J}_{123} .
\end{array}
$$

The corresponding bare MO interaction parameters, which are related to initial values of perturbations defined in Eq.(S22) in solving the RG equations, are given in Eqs.(S15-S17). The initial values of perturbations $u$ and $v$ are the same in the present model, both induced by the three-band interaction $\tilde{J}_{123}$. When $l$ approaches a sufficient large cutoff scale $l^{*}$, the generic asymptotic solutions take the form $g_{i}^{(j)} \propto\left(l^{*}-l\right)^{-\lambda_{j, i}}$. An instability takes place whenever some of the couplings diverge at a finite scale length $l^{*}$. We numerically determine the most divergent coupling when $l$ approaches $l^{*}$, which is taken to be unit, from below. For usual $3 d$-transition metals, $0<J_{H} / U<1$, we find that $g^{(1)}$ or $g_{1}^{(2)}$ dominates in the large or small $J_{H} / U$ regimes, respectively, as plotted in Figure S1(a). These two regimes correspond to the known single-band and the two-band instabilities respectively $\underline{12-14}$.

It was suggested that in addition to these one-band and two-band instabilities, there may be a new instability driven by the presence of all three bands due to the perturbation $u$ or $v$. We have checked that this instability does not occur if the initial $u$ and $v$ have the same sign as determined in the present case. We have also checked that if the initial values of $u$ and $v$ have the opposite sign, say, assuming $v / 2 \pi v_{F}=J_{H} / 6-0.02$ and $u / 2 \pi v_{F}=J_{H} / 6$ for very small $J_{H}$, there is a regime where $v$ dominates over all other perturbations as shown in Fig. S1(b). It is interesting to recall that the electron-phonon coupling, which has not been adequately considered in the present study, may result in deviations of effective perturbations away from the initial values determined in Eq.(S22). How such modification upon electron-phonon coupling takes place and whether it influences the superconductivity in the $\mathrm{K}_{2} \mathrm{Cr}_{3} \mathrm{As}_{3}$ compound deserve further investigations.

\section{The Luttinger parameters away from the condition of atomic orbital rotational symmetry}

The rotational symmetry in the interacting part of a given system is frequently assumed in literatures, by using the relationships $J_{p}=J_{H}$ and $U^{\prime}=U-J_{H}-J_{p}$ at the level of atomic orbitals. ${ }^{7}$. The relationships are adopted in plotting the phase diagram Fig. 3 in the main text. It is possible that in realistic and complicated systems this symmetry could be broken, or the above relationships could not be respected. In order to understand whether our results are still valid in this case, we consider a small deviation $\Delta U$ away from the rotational symmetry, by assuming $U^{\prime}=U+\Delta U-2 J_{H}$. It is straightforward to show that the diagonal channels are similar to the case with $\Delta U=0$ as 
given by Eq.(7) in the main text, while the model parameters appear in the Tomonaga-Luttinger Hamiltonian Eq.(8) are given by

$$
\begin{aligned}
& t_{c 1}=\frac{v_{F}}{2}+\frac{\tilde{U}_{1}}{2 \pi}, \quad t_{c 2}=\frac{v_{F}}{2}+\frac{\tilde{U}_{2}}{2 \pi}, \quad a_{c}=\frac{2 \tilde{U}_{12}}{\pi}, \quad b_{c}=\frac{2 \tilde{U}_{23}}{\pi} \\
& t_{s 1}=\frac{v_{F}}{2}-\frac{\tilde{U}_{1}}{2 \pi}, \quad t_{s 2}=\frac{v_{F}}{2}-\frac{\tilde{U}_{2}}{2 \pi}, \quad a_{s}=-\frac{\tilde{J}_{12}}{2 \pi}, \quad b_{s}=-\frac{\tilde{J}_{23}}{2 \pi} .
\end{aligned}
$$

Then, the Luttinger parameters are given by $K_{\gamma, i}=\sqrt{\frac{v_{F}}{2 \lambda_{\gamma, i}}}$, with

$$
\begin{aligned}
& \lambda_{\gamma, 1}=\frac{1}{2}\left(t_{\gamma 1}+t_{\gamma 2}\right)+\frac{1}{2}\left(b_{\gamma}+\sqrt{8 a_{\gamma}^{2}+\left(b_{\gamma}+t_{\gamma 2}-t_{\gamma 1}\right)^{2}}\right) \\
& \lambda_{\gamma, 2}=\frac{1}{2}\left(t_{\gamma 1}+t_{\gamma 2}\right)+\frac{1}{2}\left(b_{\gamma}-\sqrt{8 a_{\gamma}^{2}+\left(b_{\gamma}+t_{\gamma 2}-t_{\gamma 1}\right)^{2}}\right) \\
& \lambda_{\gamma, 3}=t_{\gamma 2}-b_{\gamma} .
\end{aligned}
$$

Explicitly, we have three pairs of charge and spin Luttinger parameters in the corresponding channels:

$$
\begin{aligned}
& K_{c, 1}=\frac{1}{\sqrt{1+G_{1}+G_{2}}}, \quad K_{c, 2}=\frac{1}{\sqrt{1+G_{1}-G_{2}}}, \quad K_{c, 3}=1 \\
& K_{s, 1}=\frac{1}{\sqrt{1-G_{1}+G_{3}}}, \quad K_{s, 2}=\frac{1}{\sqrt{1-G_{1}-G_{3}}}, \quad K_{s, 3}=1 .
\end{aligned}
$$

Where,

$$
\begin{aligned}
G_{1} & =\frac{U}{4 \pi v_{F}}+\frac{\Delta U}{12 \pi v_{F}} \\
G_{2} & =\sqrt{8\left(\frac{U+\Delta U}{6 \pi v_{F}}-\frac{5 J_{H}}{12 \pi v_{F}}\right)^{2}+\left(\frac{U+\Delta U}{12 \pi v_{F}}\right)^{2}}, \\
G_{3} & =\sqrt{8\left(\frac{J_{H}}{12 \pi v_{F}}\right)^{2}+\left(\frac{U}{12 \pi v_{F}}\right)^{2}} .
\end{aligned}
$$

The fact that the channel-" 3 " is still in the critical phase with $K_{s, 3}=K_{c, 3}=1$ is apparently due to the degeneracy of the MO bands $\nu=2$ and $\nu=3$. Based on these expressions, we find that all our results in the main text remain unchanged because the role of $\Delta U$ is to modify the value of $U$ in a simple manner.

* Electronic address: fxyong@163.com

$\dagger$ Electronic address: daijh@zju.edu.cn

${ }^{1}$ H. Zhong, X.Y. Feng, H. Chen, and J. Dai, Phys. Rev. Lett. 115, 227001 (2015).

2 There could be a prefactor $(-1)$ for the sectors $\lambda_{a}=-1$ (for $a=2,3$ ) related to the sector $\lambda_{a}=2$ (for $a=1$ ) as defined in Eq.(S9). This global prefactor can be absorded into the base definition and does not infleucne the final results.

3 H. Jiang, G. Cao, and C. Cao, Sci. Rep. 5, 16054 (2015).

${ }^{4}$ X. Wu et al., Chin. Phys. Lett. 32, 057401 (2015).

5 Y. Zhou, C. Cao, and F.C. Zhang, arXiv:1502.03928.

${ }^{6}$ X. Wu, F. Yang, C. Le, H. Fan, and J. Hu, Phys. Rev. B 92, 104511 (2015).

7 C. Castellani, C. Natoli, and J. Ranninger, Phys. Rev. B 18, 5001 (1978).

8 L. Vaugier, H. Jiang, and S. Biermann, Phys. Rev. B 86, 165105 (2012).

9 H.J. Xiang, S.-H. Mei, and M.-H. Whangbo, Phys. Rev. Lett. 100, 167207 (2008).

10 J. Solyom, Adv. Phys. 28, 201 (1979).

11 T. Giamarchi, Quantum Physics in One Dimension, Oxford University Press, Oxford, UK, 2003.

12 Y.A. Krotov, D.-H. Lee, and S.G. Louie, Phys. Rev. Lett. 78, 4245 (1997).

13 J. Gonzalez and E. Perfetto, Phys. Rev. B 72, 205406 (2005).

14 D. Carpentier and E. Orignac, Phys. Rev. B 74, 085409 (2006). 